\title{
Swift Multiwavelength Follow-up of LVC S200224ca and the Implications for Binary Black Hole Mergers
}

Klingler, N. J.; Lien, A.; Oates, S. R.; Kennea, J. A.; Evans, P. A.; Tohuvavohu, A.; Zhang, B.; Page, K. L.; Cenko, S. B.; Barthelmy, S. D.

Total number of authors:

44

Published in:

Astrophysical Journal Supplement Series

Link to article, DOI:

$10.3847 / 1538-4357 / a b d 2 c 3$

Publication date:

2021

Document Version

Publisher's PDF, also known as Version of record

Link back to DTU Orbit

Citation (APA):

Klingler, N. J., Lien, A., Oates, S. R., Kennea, J. A., Evans, P. A., Tohuvavohu, A., Zhang, B., Page, K. L., Cenko, S. B., Barthelmy, S. D., Beardmore, A. P., Bernardini, M. G., Breeveld, A. A., Brown, P. J., Burrows, D. N., Campana, S., Cusumano, G., D'Aì, A., D’Avanzo, P., ... Troja, E. (2021). Swift Multiwavelength Follow-up of LVC S200224ca and the Implications for Binary Black Hole Mergers. Astrophysical Journal Supplement Series, 907(2), [97]. https://doi.org/10.3847/1538-4357/abd2c3

\section{General rights}

Copyright and moral rights for the publications made accessible in the public portal are retained by the authors and/or other copyright owners and it is a condition of accessing publications that users recognise and abide by the legal requirements associated with these rights.

- Users may download and print one copy of any publication from the public portal for the purpose of private study or research.

- You may not further distribute the material or use it for any profit-making activity or commercial gain

- You may freely distribute the URL identifying the publication in the public portal 


\title{
Swift Multiwavelength Follow-up of LVC S200224ca and the Implications for Binary Black Hole Mergers
}

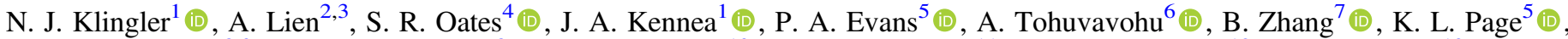 \\ S. B. Cenko ${ }^{8,9}$ (1) S. D. Barthelmy ${ }^{8}$, A. P. Beardmore ${ }^{10}$, M. G. Bernardini ${ }^{11}$ (1) A. A. Breeveld ${ }^{12}$ (1) , P. J. Brown ${ }^{13}$ (1)

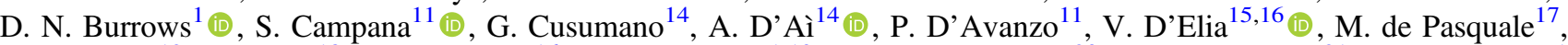 \\ S. W. K. Emery ${ }^{12}$, J. Garcia ${ }^{18}$ (D) , P. Giommi ${ }^{16}$ (), C. Gronwall ${ }^{1,19}$ (1) D. H. Hartmann ${ }^{20}$ (1) , H. A. Krimm ${ }^{21}$, N. P. M. Kuin ${ }^{12}$ (1),

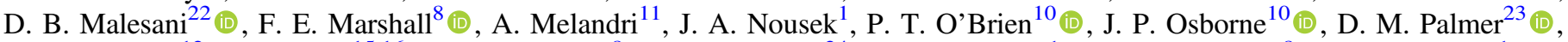

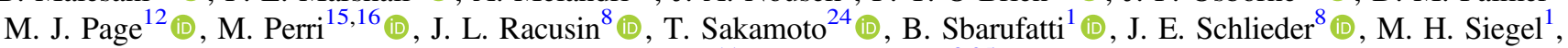 \\ G. Tagliaferri ${ }^{11}$ (D), and E. Troja ${ }^{8,25}$ (iD \\ ${ }^{1}$ Department of Astronomy and Astrophysics, The Pennsylvania State University, University Park, PA 16802, USA; njk5441@psu.edu \\ ${ }^{2}$ Center for Research and Exploration in Space Science and Technology (CRESST) and NASA Goddard Space Flight Center, Greenbelt, MD 20771, USA \\ ${ }^{3}$ Department of Physics, University of Maryland, Baltimore County, 1000 Hilltop Circle, Baltimore, MD 21250, USA \\ ${ }^{4}$ School of Physics and Astronomy, University of Birmingham, B15 2TT, UK \\ ${ }^{5}$ School of Physics and Astronomy, University of Leicester, University Road, Leicester, LE1 7RH, UK \\ ${ }^{6}$ Department of Astronomy and Astrophysics, University of Toronto, Toronto, ON, Canada \\ ${ }^{7}$ Department of Physics and Astronomy, University of Nevada, Las Vegas, NV 89154, USA \\ ${ }^{8}$ Astrophysics Science Division, NASA Goddard Space Flight Center, Greenbelt, MD 20771, USA \\ 9 Joint Space-Science Institute, Computer and Space Sciences Building, University of Maryland, College Park, MD 20742, USA \\ ${ }^{10}$ Department of Physics and Astronomy, University of Leicester, University Road, Leicester, LE1 7RH, UK \\ ${ }^{11}$ INAF-Osservatorio Astronomico di Brera, via Bianchi 46, I-23807 Merate, Italy \\ ${ }^{12}$ University College London, Mullard Space Science Laboratory, Holmbury St. Mary, Dorking, RH5 6NT, UK \\ ${ }^{13}$ George P. and Cynthia Woods Mitchell Institute for Fundamental Physics \& Astronomy, Mitchell Physics Building, Texas A. \& M. University, Department of \\ Physics and Astronomy, College Station, TX 77843, USA \\ ${ }^{14}$ INAF-IASF Palermo, via Ugo La Malfa 153, I-90146, Palermo, Italy \\ 15 INAF-Osservatorio Astronomico di Roma, via Frascati 33, I-00040 Monteporzio Catone, Italy \\ ${ }^{16}$ Space Science Data Center (SSDC)—Agenzia Spaziale Italiana (ASI), I-00133 Roma, Italy \\ ${ }^{17}$ Department of Astronomy and Space Sciences, Istanbul University, Beyzit 34119, Istanbul, Turkey \\ ${ }_{19}^{18}$ Cahill Center for Astronomy and Astrophysics, California Institute of Technology, Pasadena, CA 91125, USA \\ ${ }^{19}$ Institute for Gravitation and the Cosmos, The Pennsylvania State University, University Park, PA 16802, USA \\ ${ }^{20}$ Department of Physics and Astronomy, Clemson University, Kinard Lab of Physics, USA \\ ${ }^{21}$ National Science Foundation, Alexandria, VA 22314, USA \\ 22 DTU Space, National Space Institute, Technical University of Denmark, Elektrovej 327, DK-2800 Kongens Lyngby, Denmark \\ ${ }^{23}$ Los Alamos National Laboratory, B244, Los Alamos, NM 87545, USA \\ ${ }^{24}$ Department of Physics and Mathematics, Aoyama Gakuin University, Sagamihara, Kanagawa, 252-5258, Japan \\ ${ }^{25}$ Department of Physics and Astronomy, University of Maryland, College Park, MD 20742-4111, USA \\ Received 2020 November 12; revised 2020 December 7; accepted 2020 December 9; published 2021 February 3
}

\begin{abstract}
On 2020 February 24, during their third observing run (“O3”), the Laser Interferometer Gravitational-wave Observatory and Virgo Collaboration detected S200224ca: a candidate gravitational wave (GW) event produced by a binary black hole $(\mathrm{BBH})$ merger. This event was one of the best-localized compact binary coalescences detected in O3 (with $50 \% /$ $90 \%$ error regions of $13 / 72 \mathrm{deg}^{2}$ ), and so the Neil Gehrels Swift Observatory performed rapid near-UV/X-ray followup observations. Swift-XRT and UVOT covered approximately 79.2\% and 62.4\% (respectively) of the GW error region, making S200224ca the BBH event most thoroughly followed-up in near-UV ( $u$-band) and X-ray to date. No likely EM counterparts to the GW event were found by the Swift BAT, XRT, or UVOT, nor by other observatories. Here, we report on the results of our searches for an EM counterpart, both in the BAT data near the time of the merger, and in follow-up UVOT/XRT observations. We also discuss the upper limits we can place on EM radiation from S200224ca, as well as the implications these limits have on the physics of BBH mergers. Namely, we place a shallow upper limit on the dimensionless BH charge, $\hat{q}<1.4 \times 10^{-4}$, and an upper limit on the isotropic-equivalent energy of a blast wave $E<4.1 \times 10^{51} \mathrm{erg}$ (assuming typical GRB parameters).

Unified Astronomy Thesaurus concepts: Gravitational waves (678); High energy astrophysics (739); Black holes (162); X-ray astronomy (1810); Gamma-ray astronomy (628); Gamma-ray bursts (629); Near ultraviolet astronomy (1094)
\end{abstract}

\section{Introduction}

On 2015 September 12 the advanced Laser Interferometer Gravitational-wave Observatory (aLIGO) began its first observing run ("O1"; LIGO Scientific Collaboration et al. 2015), which ran until 2016 January 19. aLIGO consists of two interferometers located in Hanford, Washington, and Livingston, Louisiana, whose unprecedented sensitivity can detect a differential strain (over a
$4 \mathrm{~km}$ length) of less than one ten-thousandth the charge diameter of a proton. During O1, aLIGO made the first direct detections of gravitational wave $(\mathrm{GW})$ signals of astrophysical origin: GW150914, GW151226, and LVT151012 (Abbott et al. 2016a, 2016b, 2016c), marking the beginning of a new era in GW astronomy. These signals originated from the merging of binary black holes (BBHs), and thus served as an observational test of 
general relativity in the very strong field limit (in which no deviations from theory were seen).

After upgrades, aLIGO commenced its second observing run (O2) from 2016 November 30 to 2017 August 25. In August, aLIGO was augmented by the addition of the Advanced Virgo detector (a $3 \mathrm{~km}$ long interferometer located in Cascina, Italy (Acernese et al. 2015)), which collectively formed the LIGO/ Virgo Collaboration (LVC). The addition of the third detector greatly enhanced LVC's localization capabilities, decreasing the size of $\mathrm{GW}$ error regions from hundreds to tens of square degrees in the best-case scenarios. LVC also began promptly announcing GW triggers to some observatories under a memorandum of understanding, which allowed rapid followup searches. During this run, eight more GW events were detected: seven BBH mergers and a binary neutron star (BNS) merger (Abbott et al. 2019a).

The BNS merger GW170817 marked the first detection of an electromagnetic (EM) signal confidently associated with a GW event. A short gamma-ray burst (sGRB), GRB 170817A, was detected by the Fermi-GBM (Gamma-ray Burst Monitor) and INTEGRAL-SPI-ACS (SPectrometer for Integral AntiCoincidence Shield) coincident in time with the LVC trigger (Abbott et al. 2017b; Connaughton et al. 2017; Goldstein et al. 2017; Savchenko et al. 2017). ${ }^{26}$ Follow-up searches of the GRB error region resulted in the discovery of AT2017gfo (a near-infrared/optical/ultraviolet counterpart to the BNS merger produced rapidly cooling neutron-rich material ejected during the BNS merger) and the GRB 170817A afterglow whose nonthermal emission was seen in radio and X-rays (see, e.g., Abbott et al. 2017a; Evans et al. 2017; Hallinan et al. 2017; Troja et al. 2017).

LVC performed part of its third observing run (O3a) from 2019 April 1 to September 30, and resumed (O3b) from 2019 November 1 to 2020 March $27 .^{27}$ LVC also began announcing its triggers publicly and in real time through their web page. ${ }^{28}$ $\mathrm{O} 3$ resulted in 56 candidate $\mathrm{GW}$ events, including the mergers of BBHs, BNSs, and neutron star/black holes (NSBH), as well as "burst" (unmodeled) triggers, and for the first time, mergers involving objects in the mass gap range ${ }^{29}\left(\sim 3-5 M_{\odot}\right)$.

It is generally believed that the merging of isolated BBHs do not typically produce EM radiation (see, e.g., Kamble \& Kaplan 2013). However, various authors have theorized that under certain situations and/or with particular BH parameters (e.g., charged black holes, or if accreting or circumstellar material is present) $\mathrm{BBH}$ mergers may be able to produce detectable EM radiation (see, e.g., Liu et al. 2016; Loeb 2016; Perna et al. 2016; Yamazaki et al. 2016; Zhang 2016; Martin et al. 2018).

To date, there have been two purported detections of EM transients possibly associated with BBH mergers. Connaughton et al. (2016) reported a weak $1 \mathrm{~s}$ long signal above $50 \mathrm{keV}$ detected by the Fermi-GBM $0.4 \mathrm{~s}$ after GW150914 (the famous first GW signal detected, from a stellar-mass BBH). The signal's localization was consistent with that of the GW event, and had a false alarm rate (FAR) of $0.0022(2.9 \sigma)$. The authors

\footnotetext{
26 The location of the GRB was occulted by the Earth at the orbital position of Swift-BAT at the trigger time.

27 O3b was originally scheduled to proceed until 2020 April 30, but was cut short due to the COVID-19 pandemic.

28 https://gracedb.ligo.org/superevents/public/O3/

29 The mass gap refers to an apparent "gap" in the mass spectrum of NSs and BHs, as seen in the population of X-ray binaries. The heaviest NSs are $\lesssim 2.1 M_{\odot}$, and the lightest BHs are $\gtrsim 5 M_{\odot}$. See, e.g., Bailyn et al (1998), Özel et al. (2010), Özel \& Freire (2016), Abbott et al. (2019b).
}

report that the event's duration and spectra resemble a $\mathrm{sGRB}$, though with a $1 \mathrm{keV}-10 \mathrm{MeV}$ luminosity of $1.8_{-1.0}^{+1.5} \times$ $10^{49} \mathrm{erg} \mathrm{s}^{-1}$ (assuming the distance of $410 \mathrm{Mpc}$ ), which is an order of magnitude dimmer than the dimmest sGRBs (excluding GW170817A; see Wanderman \& Piran (2015)). The astrophysical origin of this signal was, however, debated by Greiner et al. (2016), and subsequently reaffirmed by Connaughton et al. (2018); see also Veres et al. (2019). The second case of possible EM radiation from a $\mathrm{BBH}$ merger was seen during $\mathrm{O} 3$ with the detection of ZTF19abanrhr (associated with AGN J124942.3+344929, a.k.a. AB 15, at $z=0.438$ ), a candidate optical counterpart to GW190521 (formerly designated S190521g; at an estimated $z=0.72 \pm 0.29$; see Abbott et al. (2020)). Graham et al. (2020) suggested that the flaring behavior seen in this optical transient is consistent with what one would expect from a kicked binary black hole merger in the accretion disk of an active galactic nucleus (AGN; McKernan et al. 2019), and they ruled out other source types. Graham et al. (2020) also predicted repeat flaring events when the kicked $\mathrm{BBH}$ remnant re-encounters the disk on timescales (in this case) of $\sim 1.6 \mathrm{yr}$, so future follow-up will shed light on the nature of this transient. If the above proposed associations with $\mathrm{BBH}$ mergers are correct, these discoveries open up exciting new prospects for the search for EM counterparts to GW events.

Another $\mathrm{O} 3 \mathrm{GW}$ trigger of interest is the $\mathrm{BBH}$ merger S200224ca. This was one of the best-localized BBH mergers, with $50 \% / 90 \%$ error regions of $13 / 72 \mathrm{deg}^{2}$ (respectively). In this paper, we will discuss the follow-up of this event performed by the Neil Gehrels Swift X-ray Telescope (XRT) and Ultraviolet/Optical Telescope (UVOT), as well as a search for prompt emission near the trigger time $\left(T_{0}\right)$ with the Burst Alert Telescope (BAT).

The paper is structured as follows. In Section 1, we describe the Swift Observatory and LVC trigger S200224ca. In Section 2, we present the results of a search for prompt emission with the BAT around the time of trigger. In Section 3, we describe Swift's follow-up procedure for GW events, both in general and for S200224ca specifically. In Section 4, we provide the details of XRT and UVOT data processing. In Section 5, we discuss the $\mathrm{X}$-ray and near-UV sources found in our follow-up searches (no confirmed EM counterparts were found). Finally, in Section 6, we discuss the implications our results have for $\mathrm{BBH}$ mergers.

\subsection{The Neil Gehrels Swift Observatory}

The Neil Gehrels Swift Observatory (Gehrels et al. 2004) is a multiwavelength space telescope in a low Earth orbit $\left(P_{\text {orbit }} \approx\right.$ 96 minutes) designed to study gamma-ray bursts (GRBs). Swift is equipped with three instruments. The Burst Alert Telescope (BAT; Barthelmy et al. 2005) consists of a coded aperture mask covering $\sim 2$ sr (or about $1 / 6$ of the sky) and operating in the 15-350 keV range. The X-ray Telescope (XRT; Burrows et al. 2005 ) is a focused X-ray imaging detector with a circular 23.6'diameter field of view (FOV), sensitive between 0.3 and $10 \mathrm{keV}$. The Ultraviolet/Optical Telescope (UVOT; Roming et al. 2005) covers a $17^{\prime} \times 17^{\prime}$ FOV. It has six filters which span the $1600-6240 \AA$ band, and a white filter sensitive from 1600 to $8000 \AA$, as well as UV and optical grisms for spectroscopy.

When the BAT triggers on a GRB, Swift calculates its position to an accuracy of $1^{\prime}-3^{\prime}$, then autonomously and promptly slews to the burst (usually within 1-3 minutes, assuming that the burst is not located within Swift's regions of Sun, Earth, or Moon 
Table 1

LVC S200224ca Details

\begin{tabular}{lc}
\hline \hline Parameter & Value \\
\hline Trigger date & $2020-02-24$ \\
Trigger time & $22: 22: 34.390 \mathrm{UT}$ \\
Trigger type & Compact binary coalescence \\
Detectors & $\mathrm{H} 1, \mathrm{~L} 1, \mathrm{~V} 1 ;($ all 3$)$ \\
Estimated distance & $1575 \pm 322 \mathrm{Mpc}$ \\
$P_{\mathrm{BBH}}$ & 0.999966 \\
$P_{\mathrm{MG}}$ & 0.0 \\
$P_{\mathrm{NSBH}}$ & 0.0 \\
$P_{\mathrm{BNS}}$ & 0.0 \\
$P_{\mathrm{Terrestrial}}$ & $3.3852 \times 10^{-5}$ \\
False alarm rate & $1.605 \times 10^{-11} \mathrm{~Hz}(1 / 1974 \mathrm{yr})$ \\
\hline Swift follow-up start time & $T_{0}+336.6 \mathrm{minutes}$ \\
Fields observed & 672 \\
TargetID range & $703157-7032252$ \\
\hline
\end{tabular}

Note. $P_{\text {(event) }}$ is the probability the event was produced by a binary black hole $(\mathrm{BBH})$, mass gap object $(\mathrm{MG})$, neutron star + black hole $(\mathrm{NSBH})$, binary neutron star (BNS), or of terrestrial origin.

avoidance at trigger time). The afterglow is then observed by the XRT and UVOT, which provide arcsecond-scale localizations.

Although initially designed to study GRBs, Swift's ability to quickly respond to and observe targets of opportunity (TOOs) has enabled it to become a "first responder" to transient astronomical events of all types, including the search for EM counterparts to GW events. Swift has performed rapid follow-up of observations during both $\mathrm{O} 2$ and $\mathrm{O} 3$, the results of which have been reported by Evans et al. (2016a, 2016b, 2016c, 2017, 2019), Klingler et al. (2019), Page et al. (2020), and S. R. Oates et al. (2020, in preparation).

\subsection{Trigger $S 200224 c a$}

On 2020 February 24 at 22:23:34 UT, all three LVC detectors triggered on S200224ca (LIGO Scientific Collaboration \& Virgo Collaboration 2020a). The event had a low FAR of $1.605 \times$ $10^{-11} \mathrm{~Hz}$ (or roughly once every $1974 \mathrm{yr}$ ) and a low probability of being of terrestrial origin $\left(P_{\text {Terrestrial }}=3.3852 \times 10^{-5}\right)$. The candidate $\mathrm{GW}$ event was determined to have a very high probability of being produced by a binary black hole merger (i.e., probability $P_{\mathrm{BBH}} \approx 1.0$ ) at an estimated distance $d=1575 \pm 322$ Mpc. Additional trigger details are listed in Table 1. The event was very well-localized, with initial $50 \%$ and $90 \%$ probability areas corresponding to 17 and $69 \mathrm{deg}^{2}$, respectively (these were subsequently refined to 13 and $72 \mathrm{deg}^{2}$ by the LIGO Scientific Collaboration \& Virgo Collaboration (2020b)).

\section{Search for a Counterpart in BAT Data near $\boldsymbol{T}_{0}$}

We performed a search for a counterpart in the BAT data within $T_{0} \pm 100 \mathrm{~s}$. Specifically, we searched for potential detections in the BAT raw light curves and images created by the BAT survey data and the available BAT event data. During O3, Swift had begun testing the BAT Gamma-ray Urgent Archiver for Novel Opportunities (GUANO) system (Tohuvavohu et al. 2020). GUANO is a system which proactively downlinks BAT event data from the spacecraft (which are otherwise discarded in the absence of a BAT trigger, due to telemetry limitations) upon receiving notice of a relevant astronomical event detected by other facilities. The retrieval of
BAT event data allows for much more sensitive searches for prompt (sGRB-like) emission from compact binary coalescence (CBC) events, and increases the number of possible co-detections of GW and EM radiation. Unfortunately, for S200224ca, only event data from $T_{0}+29.042 \mathrm{~s}$ to $T_{0}+45.176 \mathrm{~s}$ were retrieved from BAT's ring buffer, due to the GUANO command reaching the Swift spacecraft a little too late, ${ }^{30}$ at $\sim T_{0}+28$ minutes instead of the median $\sim T_{0}+16$ minutes for $\mathrm{GW}$ triggers.

Our analysis of event data and survey data utilized the standard HEASoft/BAT tools. Specifically, batgrbproduct was used for the event data analysis, batsurvey was used for survey data analysis, and batcelldetect was used to search for potential detections in images. Analysis of the BAT data was also reported via GCN circular by Barthelmy et al. (2020).

Swift was slewing from $\sim T_{0}-23 \mathrm{~s}$ to $\sim T_{0}+180 \mathrm{~s}$. Figure 1 shows the BAT raw light curve (panel (a)) and how the BAT FOV coverage of the LVC probability region changes as a function of time. As BAT is a coded aperture mask instrument, the sensitivity is highest at the center of the FOV, where the coding fraction is $\sim 100 \%$. The sensitivity decreases toward the edge of the $\mathrm{FOV}$, as the coding fraction decreases to zero. ${ }^{31}$ The integrated LVC localization probability inside the BAT FOV ( $>10 \%$ partial coding fraction) is shown in panel (b). In addition, BAT retains decreased (but still significant) sensitivity to rate increases for gamma-ray events outside of its FOV. In panel (c), we also show the integrated LVC localization probability that is outside the BAT FOV but above the Earth limb. At $T_{0}$, the $\mathrm{S} 200224 \mathrm{ca}$ integrated probability inside the BAT FOV is 0.8855 , and the probability outside of BAT FOV but above the Earth limb is 0.1145 . The BAT FOV at $T_{0}$ and S200224ca localization are also overlaid in the right panel of Figure 2.

Within our search time window of $T_{0} \pm 100 \mathrm{~s}$, the BAT raw light curves in different time bins (64 ms, $1 \mathrm{~s}, 1.6 \mathrm{~s})$ and energy bands $(15-25,25-50,50-100$, and $100-350 \mathrm{keV})$ show no significant detections with signal-to-noise ratio $\gtrsim 5 \sigma$. We estimate the flux upper limit using the $1.6 \mathrm{~s}$ light curve $(15-350 \mathrm{keV})$. Within $T_{0} \pm 100 \mathrm{~s}$, the light curve has a $5 \sigma$ standard deviation of $\sim 411$ count $\mathrm{s}^{-1}$, which corresponds to a $5 \sigma$ flux upper limit of $\sim 4.76 \times 10^{-7} \mathrm{erg} \mathrm{cm}^{-2} \mathrm{~s}^{-1}$ (in $15-350 \mathrm{keV}$ ). The conversion from instrumental count to energy flux uses the method developed by Lien et al. (2014), and assumes a typical BAT-detected short GRB spectrum (a simple power-law model with a photon index of 1.32; see Lien et al. (2016)) and an instrumental response for a partial coding fraction of $\sim 0.19$, which is the average partial coding fraction of the LVC region inside the BAT FOV at $T_{0}$.

The event data closest to $T_{0}$ only cover the time range of $T_{0}+29.04 \mathrm{~s}$ to $T_{0}+45.18 \mathrm{~s}$. No significant detections $(\gtrsim 5 \sigma)$ are found in the image $(15-150 \mathrm{keV})$ made using these event data. Because the time interval covers a spacecraft slew, this image is made by co-adding subimages of $0.5 \mathrm{~s}$ intervals.

BAT collects continuous survey data (i.e., data binned in intervals of $\sim 300 \mathrm{~s}$ ) while the spacecraft is in pointing mode. Because of the spacecraft slews, no survey data are available at $T_{0}$. The closest available survey data cover time ranges of

\footnotetext{
30 This was due to a long request queue in the Tracking and Data Relay Satellite System (TDRSS) and the unavailability of ground station passes shortly after $T_{0}$.

31 For more details on the coded aperture mask instrument and the definition of the coding fraction, see the Swift BAT Software Guide, http://swift.gsfc.nasa. gov/analysis/bat_swguide_v6_3.pdf.
} 


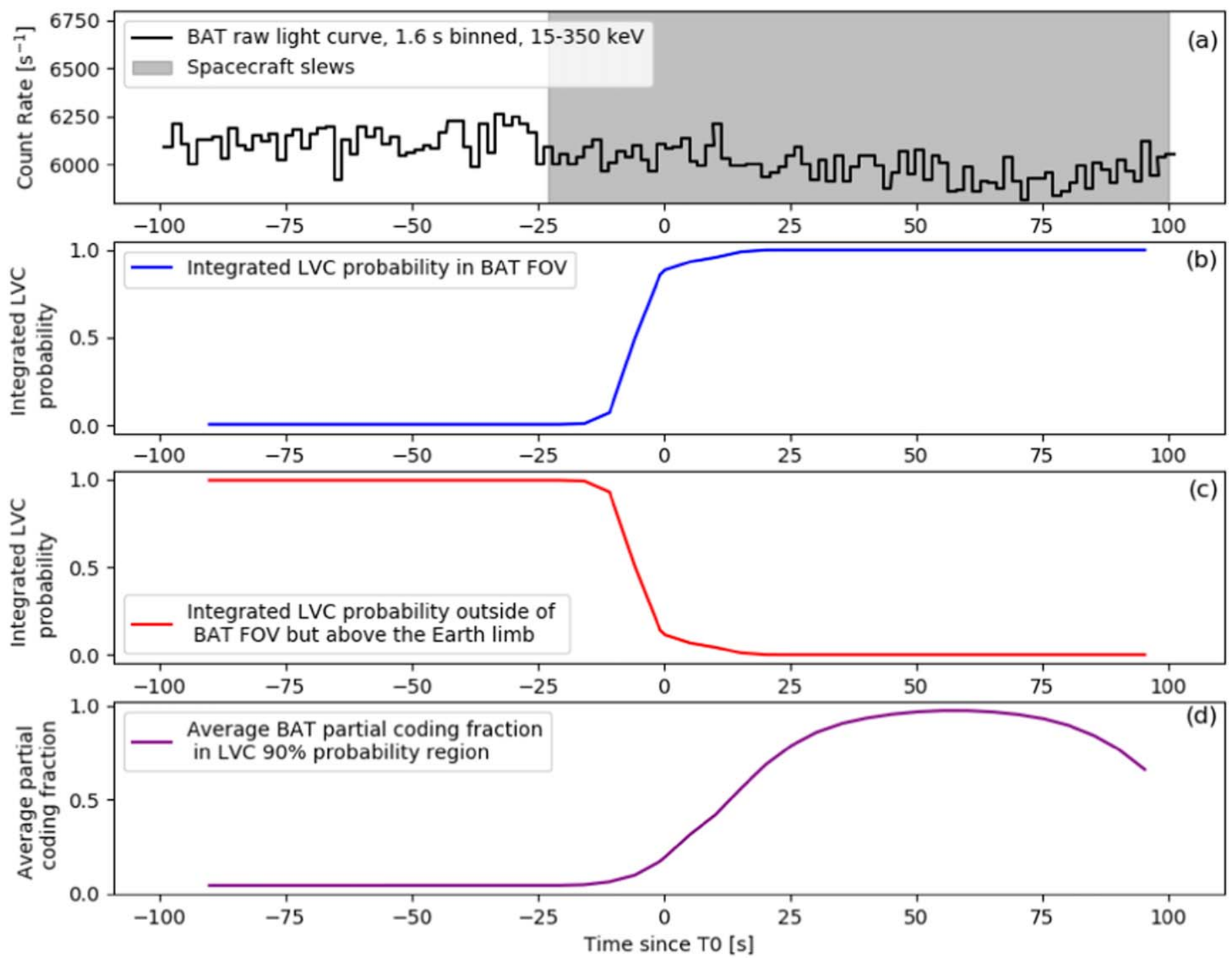

Figure 1. Panel (a): BAT raw light curve (15-350 keV) consisting of $1.6 \mathrm{~s}$ bins. Gray area marks the time interval of spacecraft slewing. Panel (b): the integrated LVC localization probability inside the BAT FOV ( $>10 \%$ partial coding fraction). Panel (c): the integrated LVC localization probability outside the BAT FOV but above the Earth limb. Panel (d): the average BAT partial coding fraction inside the $90 \%$ LVC localization probability region. The integrated LVC probability and average partial coding fraction plots correspond to the LALInference map (i.e., the most recent skymap for this event).

$T_{0}-144.96 \mathrm{~s}$ to $T_{0}-21.96 \mathrm{~s}$, and $T_{0}+180.02 \mathrm{~s}$ to $T_{0}+480.04 \mathrm{~s}$. Again, no significant detections are found in images created by survey data in these two time intervals.

\section{Swift GW Follow-up}

Swift's criteria and procedure for performing pointed follow-up searches of GW error regions (with UVOT/XRT) have been described in detail by Evans et al. (2016b, 2016c), and subsequent improvements have been described by Klingler et al. (2019) and Page et al. (2020), so only an abridged description will be provided here, in Section 3.1. In Section 3.2, we describe the observing plan for S200224ca.

\subsection{General Follow-up Procedure}

Upon notification of a new LVC trigger, our GW planning pipeline determines whether our follow-up criteria are met. Factors that determine this include the type of event (e.g., CBC or burst event), the probability that the $\mathrm{GW}$ event involved a neutron star $P_{\mathrm{NS}}$, the event's estimated FAR, the probability the signal was of terrestrial origin $P_{\text {Terrestrial }}$, and the percentage of the probability region that Swift-XRT can observe within $24 \mathrm{hr}$ $P_{24}$ hr (the full follow-up criteria are described by Page et al. (2020)).

When the 3D LVC probability map is received, it is automatically convolved with the 2MASS Photometric Redshift Galaxy Catalog (2MPZ; Bilicki et al. 2014), using the method described by Evans et al. (2016b, 2019). This approach accounts for galaxy catalog (in)completeness. For events at large distances where galaxy catalogs are highly incomplete, as is the case for $200224 \mathrm{ca}$, the convolved skymap is only minimally altered from the original LVC map. We use this reweighted probability map to create a prioritized observing plan.

\subsection{S200224ca Follow-up}

Upon receiving automated notice of the trigger, our LVC processing pipeline calculated $P_{24 \mathrm{hr}}=0.66$, which met our criteria for follow-up. A tiling plan was created and uploaded to Swift at the next available ground station pass, and Swift began observations at $T_{0}+22 \mathrm{ks}$. Six hundred and seventy-two fields 

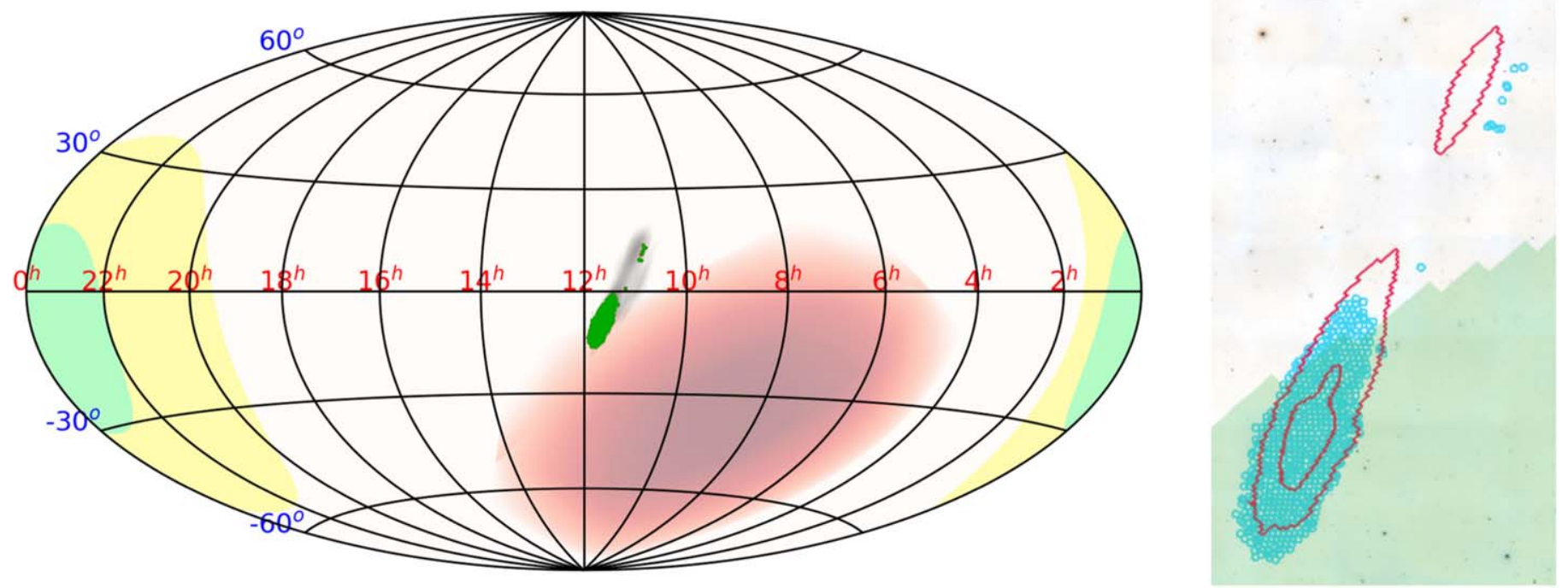

Figure 2. Left: updated skymap of the S200224ca localization (from the LALInference pipeline; shown by the gray area). The area covered by the XRT follow-up is highlighted in dark green, and the region of sky covered by BAT's coded aperture mask at the time of trigger $\left(T_{0}\right)$ is highlighted in red. Yellow and light green areas represent Swift's areas of Sun and Moon avoidance at $T_{0}$. Right: zoomed-in image of the central regions of the S200224ca localization, overlaid on a DSS image (produced by treasuremap.space; see Wyatt et al. (2020)). The 50\%/90\% contours are enclosed in red, the XRT fields are circled in blue, and the region of sky covered by BAT (i.e., $>10 \%$ coding fraction) at $T_{0}$ is highlighted in green. The XRT fields outside of the northern (50\%) lobe were due to Swift performing observations prior to the release of the updated (LALInference) skymap.

were observed (the UVOT filter wheel was in "blocked" mode for two of these fields, due to the presence of bright stars). Swift completed the phase $1(80 \mathrm{~s})$ tilings at $T_{0}+196.5 \mathrm{ks}$, but the planned phase $2(500 \mathrm{~s})$ tilings were not carried out, due to the announcement of the following GW event, S200225q, whose follow-up took priority. The XRT covered $61.2 \mathrm{deg}^{2}$ (taking into account tiles with overlapping regions), corresponding to $79.2 \%$ of the GW probability region (using the most recent LALInference skymap). ${ }^{32}$ In Figure 2, we show the S200224ca localization with the area covered by the XRT overlaid, and in Figure 3, we show the percentage of the S200224ca covered by the XRT as a function of time. The UVOT performed followup in the $u$-band (near-UV) and covered $46.2 \mathrm{deg}^{2}$ (due to its FOV being smaller than the XRT's), corresponding to $62.4 \%$ of the raw probability region (see Figure 4).

\section{Data Processing}

\subsection{XRT Data Processing}

Upon being downlinked, XRT data were automatically processed at the United Kingdom Swift Data Science Centre (UKSSDC) at the University of Leicester, using HEASoft v6.26.1 and the latest CALDB available at the time of processing. The xrtpipeline tool is run, which applies all necessary calibrations, filtering, and corrections. ${ }^{33}$ Images and exposure maps of each observation are also created.

The goals of the subsequent data processing are as follows: (1) search for sources, (2) characterize them, and (3) identify potential counterparts to the GW trigger.

The source detection pipeline is that used in the production of the 2nd Swift X-ray Point Source Catalog (2SXPS), which is described in detail by Evans et al. (2020). This is an iterative process that utilizes sliding-cell source detection, background modeling, PSF fitting, and a likelihood test to detect and

\footnotetext{
32 See https://gracedb.ligo.org/superevents/S200224ca/.

33 For more details, see http://www.swift.ac.uk/analysis/ and https://heasarc. gsfc.nasa.gov/ftools/caldb/help/xrtpipeline.html.
}

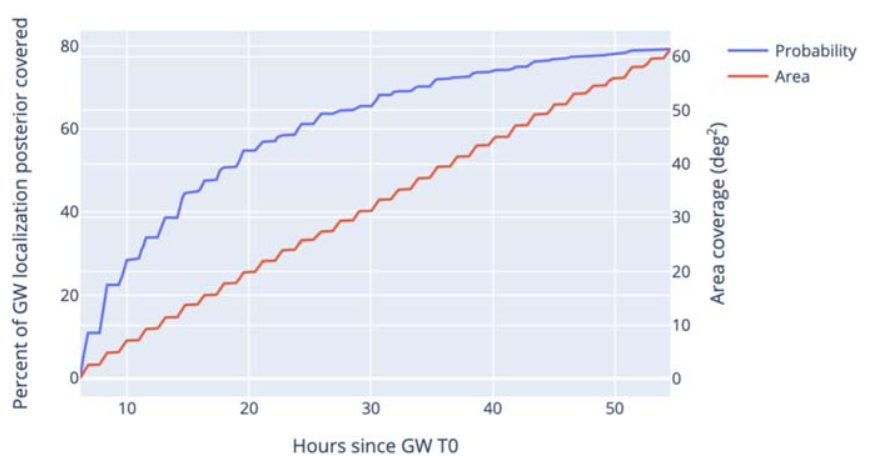

Figure 3. Percentage of the S200224ca GW localization (blue) and raw area (red) covered by the XRT as a function of time (using the latest LALInference skymap from treasuremap.space; see Wyatt et al. (2020)). The step-like behavior of the plot results from a combination of Swift's low-Earth orbit and Earth-limb pointing constraints, which causes any given region of sky to be only visible for (at most) roughly half of the $\approx 96$ minutes orbit.

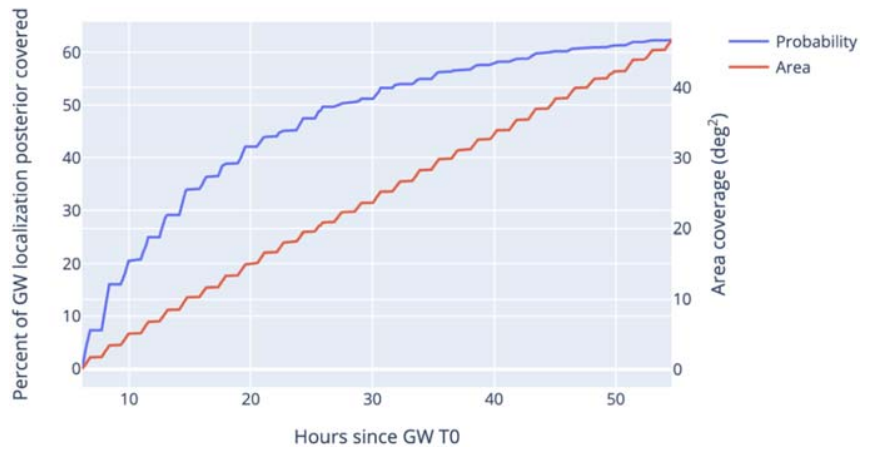

Figure 4. Percentage of the S200224ca GW localization (blue) and raw area (red) covered by the UVOT as a function of time (using the latest LALInference skymap from treasuremap.space; see Wyatt et al. (2020)).

localize sources. Each detected source is assigned a quality flag, which describes the probability of the source being a spurious detection. Sources flagged as "good" have a $0.3 \%$ or 
less chance of being spurious (or false positive; FP), "reasonable" sources have a 7\% FP rate, and "poor" sources have up to a 35\% FP rate. All sources detected are manually inspected for spurious detections that may arise from optical loading, stray light, extended emission, and/or thermal noise from the XRT detector getting too hot.

For source characterization, the sources are assigned a rank that indicates how likely each is to be an EM counterpart to a GW trigger, as defined by Evans et al. (2016a) (this is same ranking system used during Swift follow-up of O2 triggers; see Klingler et al. (2019)). Rank 1 is assigned to counterpart candidates. Sources of this rank are: (1) uncataloged and at least $5 \sigma$ above the $3 \sigma$ upper limit from the ROSAT All-Sky Survey (RASS) or 2SXPS, or (2) a known X-ray source that is $5 \sigma$ above its cataloged flux. ${ }^{34}$ Rank 1 sources must also lie near (within $200 \mathrm{kpc}$ in projection of) a known galaxy (assuming the source is at the distance of that galaxy). Rank 2 is assigned to "interesting" sources. These sources each fall into one of two types: (1) uncataloged sources that are either at least $3 \sigma$ above the $3 \sigma$ upper limit from the RASS/2SXPS or are fading; or (2) known X-ray sources at least $3 \sigma$ above their cataloged flux. Unlike afterglow candidates, an interesting source need not be near a known galaxy. Rank 3 is assigned to X-ray sources not previously cataloged, and which were not bright enough to suggest a transient nature. Rank 4 is assigned to known X-ray sources whose flux is consistent with (or below) that from their previous observations.

Light curves are produced for each detected source using all follow-up data, and sources exhibiting evidence of fading are flagged.

\subsection{UVOT Data Processing}

Analysis of the UVOT data is performed via the UVOT GW processing pipeline, for which we provide a brief overview here (for full details, see S. R. Oates et al. (2020, in preparation)). The pipeline searches the UVOT sky images to identify new transient sources that might be the counterpart to the GW event. For each observation, the HEASoft ftool utility uvotdetect (based on SExtractor; see Bertin \& Arnouts (1996)) is run for that exposure to search for sources. All sources found are then run through a series of checks to determine if they are previously cataloged (known) sources, extended sources, sources due to image artifacts, or minor planets. Sources that pass all quality-control checks are labeled as Q0 or Q1, depending on their magnitude. Sources dimmer than a magnitude of 18.9 (the typical sensitivity limit for tiling observations) are assigned as Q1. The pipeline reliably finds new sources if they are isolated from existing sources and not affected by defects in the UVOT images (artifacts) caused by very bright sources. Small images (thumbnails) are produced for each Q0 and Q1 source, and also for all nearby galaxies reported in the GLADE Catalog (Dálya et al. 2018) whose positions were observed by UVOT. This enables rapid manual inspection to evaluate the reliability of possible UVOT counterparts. Also, for every Q0/Q1 UVOT source found,

\footnotetext{
34 The archival upper limits / count rates (i.e., from RASS, or 2SXPS when available) for both criteria were not derived from XRT data; they have been converted to equivalent XRT (PC mode) $0.3-10 \mathrm{keV}$ count rates using PIMMS (Portable Interactive Multi-Mission Simulator), assuming a typical AGN spectrum (absorbing hydrogen column density $N_{\mathrm{H}}=3 \times 10^{20} \mathrm{~cm}^{-2}$, and photon index $\Gamma=1.7)$. The peak source fluxes were also obtained by converting from the peak count rates when assuming a typical AGN spectrum with the abovementioned parameters.
}

the XRT analysis is automatically repeated to determine flux measurements at the UVOT source position, which is more sensitive than a blind search. If the $3 \sigma$ lower limit on the XRT count rate is $>0$, then an X-ray counterpart to the UVOT source is reported as detected; otherwise, the $3 \sigma \mathrm{UL}$ is reported. In the case of S200224ca, only upper limits were found.

\section{S200224ca Follow-up Results}

No confirmed or likely counterparts to S200224ca were detected by Swift nor by other observatories. Below, we describe the (uncataloged) XRT sources and the near-UV sources detected and flagged by the XRT/UVOT pipelines. $\mathrm{XRT}$ count rates provided were corrected for vignetting effects.

\subsection{X-Ray Sources}

Swift-XRT detected eight sources during the follow-up of S200224ca. Two were rank 3 (uncataloged X-ray sources, though not significantly above the RASS upper limit), and six were rank 4 (known X-ray sources whose fluxes were consistent within $3 \sigma$ of their cataloged values). Details for each source are listed in Table 2. Source 9 had a detection flag of "reasonable," and the rest had detection flags of "good."

The two rank 3 sources were Sources 5 and 9. Source 5 was first detected during Observation 07032074001, which began on 2020 February 26 at 15:56:43 UT. During the 82 s exposure, the source was observed to have a count rate of $0.09_{-0.04}^{+0.05} \mathrm{ct} \mathrm{s}^{-1}$. It was observed again during Observation 07032138001, which began at 2020 February 26 at 20:38:59 UT, during which the count rate was $0.02_{-0.01}^{+0.03}$, thus exhibiting evidence of fading at $1.3 \sigma$. Source 5 was reobserved for $1.7 \mathrm{ks}$ on 2020 May 24, after which it was found to have shown no evidence of fading after six months (see the light curve in Figure 5). The nearest cataloged source is quasar J113530.8-124219, located 10.6" from the XRT position (though it is outside the $90 \%$ positional uncertainty radius of $6.3^{\prime \prime}$.

Source 9 was detected during Observation 07032038001, which began on 2020 February 26 at 12:57:23 UT $\left(\approx T_{0}+139 \mathrm{ks}\right)$. During the $75 \mathrm{~s}$ exposure, five counts were detected (within a 30" aperture radius), yielding a count rate of $0.09_{-0.04}^{+0.05} \mathrm{ct} \mathrm{s}^{-1}$. The target's location was reobserved a few minutes later at 13:00:53 (because the target resided in the overlapping region between two planned pointings, the second of which was Observation 07032040001 ), but no counts were detected during the $77 \mathrm{~s}$ exposure. The XRT images and exposure maps of Observations 07032038001 and 07032040001 are shown in Figure 6. In the first observation (07032038001), Source 9's position did not land within the UVOT FOV. In the second observation (07032040001), Source 9's position resided in the UVOT's FOV (at the very edge) but no near-UV counterpart was seen.

Source 9's position was reobserved four times between May and June 2020 (totaling $8.2 \mathrm{ks}$; targetID 7400091), but no counts were detected within a $30^{\prime \prime}$ aperture, yielding a count rate $<1.2 \times 10^{-4} \mathrm{ct} \mathrm{s}^{-1}$. An $8.2 \mathrm{ks}$ exposure corresponds to a detection limit of approximately $7.5 \times 10^{-14} \mathrm{erg} \mathrm{cm}^{-2} \mathrm{~s}^{-1}$ at $50 \%$ confidence (or $1.5 \times 10^{-13} \mathrm{erg} \mathrm{cm}^{-2} \mathrm{~s}^{-1}$ at $90 \%$ confidence; see the XRT sensitivity curve in Figure 6 of Evans et al. (2015)). No near-UV counterpart was seen in any of the corresponding UVOT images. There are no cataloged SIMBAD sources within $18^{\prime \prime}$ of this source. The nearest VizieR cataloged source is SDSS J113719.13-044407.7, located $8^{\prime \prime}$ from the XRT position (though it is outside the $90 \%$ positional uncertainty radius of $6.2^{\prime \prime}$ ). 
Table 2

Detected XRT Sources

\begin{tabular}{lccccccccccccc}
\hline \hline$\#$ & Rank & $\begin{array}{c}\text { R.A./Decl. } \\
(\mathrm{J} 2000)\end{array}$ & $\begin{array}{c}\text { Err. } \\
(90 \%) \\
(\mathrm{arcsec})\end{array}$ & $\begin{array}{c}\text { Exposure } \\
(\mathrm{s})\end{array}$ & $\begin{array}{c}\text { Peak } \\
\text { Rate } \\
\left(\mathrm{ct} \mathrm{s}^{-1}\right)\end{array}$ & $\begin{array}{c}\text { Peak } \\
F_{-12} \\
(\mathrm{cgs})\end{array}$ & $\begin{array}{c}\text { Fading } \\
(\sigma)\end{array}$ & $\begin{array}{c}D_{\mathrm{LVC}} \\
(\mathrm{Mpc})\end{array}$ & $\begin{array}{c}\text { Peak } \\
L_{44} \\
\left(\mathrm{erg} \mathrm{s} \mathrm{s}^{-1}\right)\end{array}$ & $\begin{array}{c}F_{\text {Obs }}: F_{\text {Cat }} \\
(\sigma)\end{array}$ & $\begin{array}{c}\text { G/2/S } \\
\text { Comp. }\end{array}$ & $\begin{array}{c}\text { Gal. } \\
\text { Comp }\end{array}$ \\
\hline 5 & 3 & $173.8795-12.7028$ & 6.3 & 1880 & $0.09_{-0.04}^{+0.05}$ & $3.7_{-1.6}^{+2.1}$ & 0 & $1391 \pm 363$ & $8.5 \pm 1.8$ & 0 & $0 / 0 / 0$ & $4.7 \%$ \\
9 & 3 & $174.3296-4.7332$ & 6.2 & 7032 & $0.09_{-0.04}^{+0.05}$ & $3.7_{-1.6}^{+2.1}$ & 2.3 & $1428 \pm 298$ & $9.1 \pm 2.0$ & 2.9 & $0 / 0 / 0$ & $3.6 \%$ \\
\hline 1 & 4 & $175.5795-14.3774$ & 5.2 & 80 & $0.09_{-0.03}^{+0.05}$ & $3.9_{-1.3}^{+2.2}$ & $\ldots$ & $1551 \pm 334$ & $11 \pm 2.2$ & 1.4 & $0 / 2 / 1$ & $3.7 \%$ \\
3 & 4 & $173.9797-11.7069$ & 5.9 & 65 & $0.07_{-0.03}^{+0.03}$ & $2.9_{-1.2}^{+2.1}$ & $\ldots$ & $1470 \pm 335$ & $7.5 \pm 1.8$ & 0 & $0 / 2 / 1$ & $3.9 \%$ \\
4 & 4 & $162.5324+11.5419$ & 4.5 & 95 & $0.21_{-0.07}^{+0.08}$ & $8.9_{-3.0}^{+3.4}$ & 1.4 & $1587 \pm 347$ & $3.7 \pm 0.6$ & 2.8 & $0 / 1 / 1$ & $12.5 \%$ \\
7 & 4 & $175.4238-14.1306$ & 5.2 & 137 & $0.09_{-0.04}^{+0.05}$ & $3.8_{-1.7}^{+2.1}$ & 0 & $1544 \pm 332$ & $1.1 \pm 0.2$ & 0 & $0 / 1 / 1$ & $3.7 \%$ \\
8 & 4 & $171.4663-7.7071$ & 5.1 & 313 & $0.06_{-0.02}^{+0.02}$ & $2.5_{-0.6}^{+0.8}$ & $\ldots$ & $1173 \pm 394$ & $4.1 \pm 0.5$ & 2.8 & $0 / 1 / 1$ & $6.3 \%$ \\
10 & 4 & $178.0148-11.3727$ & 4.7 & 155 & $0.10_{-0.04}^{+0.05}$ & $4.3_{-1.7}^{+2.2}$ & 0 & $1432 \pm 394$ & $10.4 \pm 2.0$ & 0 & $0 / 1 / 1$ & $5.0 \%$ \\
\hline
\end{tabular}

Note. Details of the sources detected. The following are listed: source number (sources were numbered in the order of detection; missing numbers were sources confirmed to be spurious detections), source rank, position and 90\% uncertainty, total XRT exposure (corrected for vignetting effects), peak count rate (0.3-10 keV), peak flux $F_{-12}$ (in units of $10^{-12} \mathrm{erg} \mathrm{cm}^{-2} \mathrm{~s}^{-1}$ ), the significance of any fading (“...” notes sources observed once), the LVC estimated distance at the source's position $D_{\mathrm{LVC}}$, and the peak X-ray luminosity $L_{44}$ (if the source was located at $D_{\mathrm{LVC}}$, in units of $10^{44} \mathrm{erg} \mathrm{s}^{-1}$ ). $F_{\mathrm{Obs}}: F_{\mathrm{Cat}}$ shows the significance of the ratio of the observed flux to the cataloged flux; for uncataloged (rank 3) sources, the XRT-equivalent of the RASS upper limit was used instead. The G/2/S column notes whether the source's position (and uncertainty) are consistent with any known GWGC/2MPZ galaxies, 2MASS sources, or SIMBAD sources. Gal. Comp. is the completeness of the galaxy catalog along the line of sight to the source.

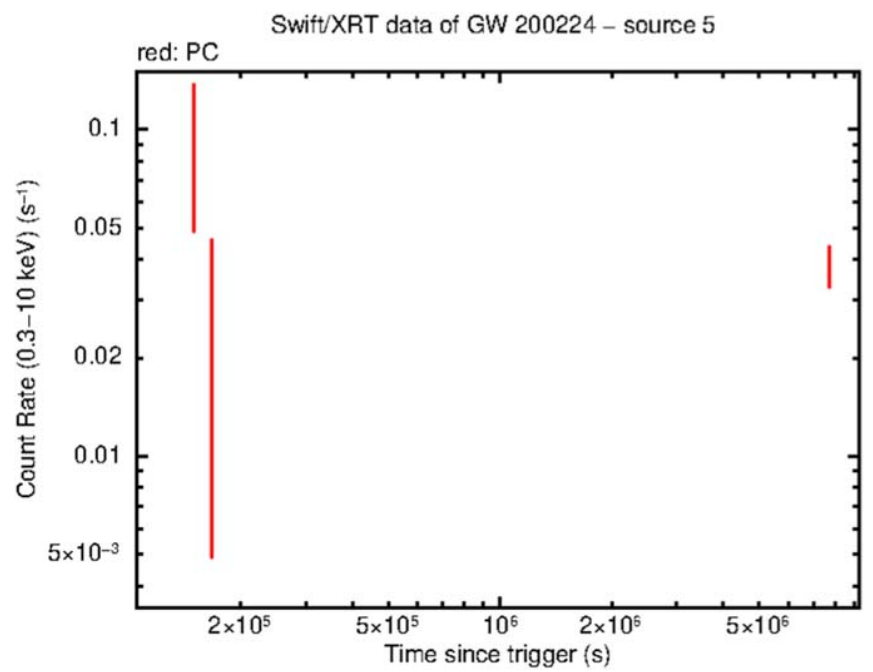

Figure 5. XRT light curve of Source 5.

The detection of Source 9 in ObsID 7032038001 but not in ObsID 7032040001 (which occurred four minutes later) is puzzling. Assuming that Source 9's count rate is Poissonian (and that it did not change noticeably over the course of those four minutes), the probability of detecting zero counts during the second (77.4 s) exposure is low: 0.000943. Considering this, it is possible that the detection of Source 9 in the first observation was a false positive resulting from noise fluctuations, as its detection flag was only "reasonable" (sources of this flag have a $7 \%$ false positive rate). Even if Source 9 was real, such fading over the course of minutes at $T_{0}+139 \mathrm{ks}$ is not consistent with power-law fading observed in SGRBs (see, e.g., Fong et al. (2015), although BBH mergers are not expected to produce sGRBs under typical circumstances).

\subsection{Near-UV Sources}

The UVOT GW processing pipeline produced 1654 thumbnails from the 670 observed fields. The majority of these thumbnails were of galaxies, but 17 and 65 thumbnails were produced for Q0 and Q1 sources, respectively. Of these, manual inspection ruled out the majority of these sources, but 1 Q0 source and 2 Q1 sources were still of interest after manual inspection. S. R. Oates et al. (2020, in preparation) provide additional discussion of these sources, as well as a table listing the photometry.

Q0_src47 was found at R.A., decl. (J2000) $=176.58794$, -11.46508 with an estimated uncertainty of $0.8^{\prime \prime}$ (radius, $90 \%$ confidence), starting at $T_{0}+63.77 \mathrm{ks}$. The initial $u$-band magnitude $^{35}$ was $u=18.62 \pm 0.23$ (19.64 mag. $\mathrm{AB}$ ), giving a $4.8 \sigma$ detection. This source was announced to the astronomical community via Breeveld et al. (2020). No follow-up was reported by other facilities. In the Pan-STARRS catalog, a point-like source was found (objID 94241765879572233) at a position consistent with this $\mathrm{Q} 0$ source with $(\mathrm{AB})$ magnitudes $z=20.74 \pm 0.04, \quad i=21.59 \pm 0.23$. Pan-STARRS did not detect the source in the $g$ - or $r$-bands, with limiting magnitudes $(5 \sigma, \mathrm{AB})>23.3$ and $>23.2$, respectively. Q0_src47 was not detected in any filter in the second UVOT visit $(u>19.6)$ occurring at $T_{0}+3.18$ days (or 2.5 days after the first detection); thus, the target faded by $\Delta u>0.4$ mag day $^{-1}$. The observed rapid fading rules out slowly evolving transients such as such as SNe (Wheeler \& Harkness 1990; GalYam 2012), tidal disruption events (van Velzen et al. 2020), and AGN (MacLeod et al. 2012; Smith et al. 2018), but faster evolving transients such as GRBs (Sari et al. 1998), kilonovae (Metzger 2019), and flare stars (Osten et al. 2010; Schmidt et al. 2014) cannot be excluded. Archival observations by PanSTARRS show that Q0_src47 is point-like and very red. Its brightness increases toward red wavelengths and it is at least two magnitudes fainter in $g$ and $r$ compared to $i, z$, and $Y$. Considering the target's point-like nature, red quiescent color, and rapid fading, it is likely that what UVOT detected was a flare from a low-mass star (for further analysis of this source, see S. R. Oates et al. (2020, in preparation)). This source was reobserved again on 2020 June $30\left(T_{0}+127\right.$ days), but was not detected $(u>20.8)$.

\footnotetext{
35 The UVOT source magnitudes listed in this work correspond to the Vega magnitude system. Absolute magnitudes (AB) are provided in parentheses.
} 


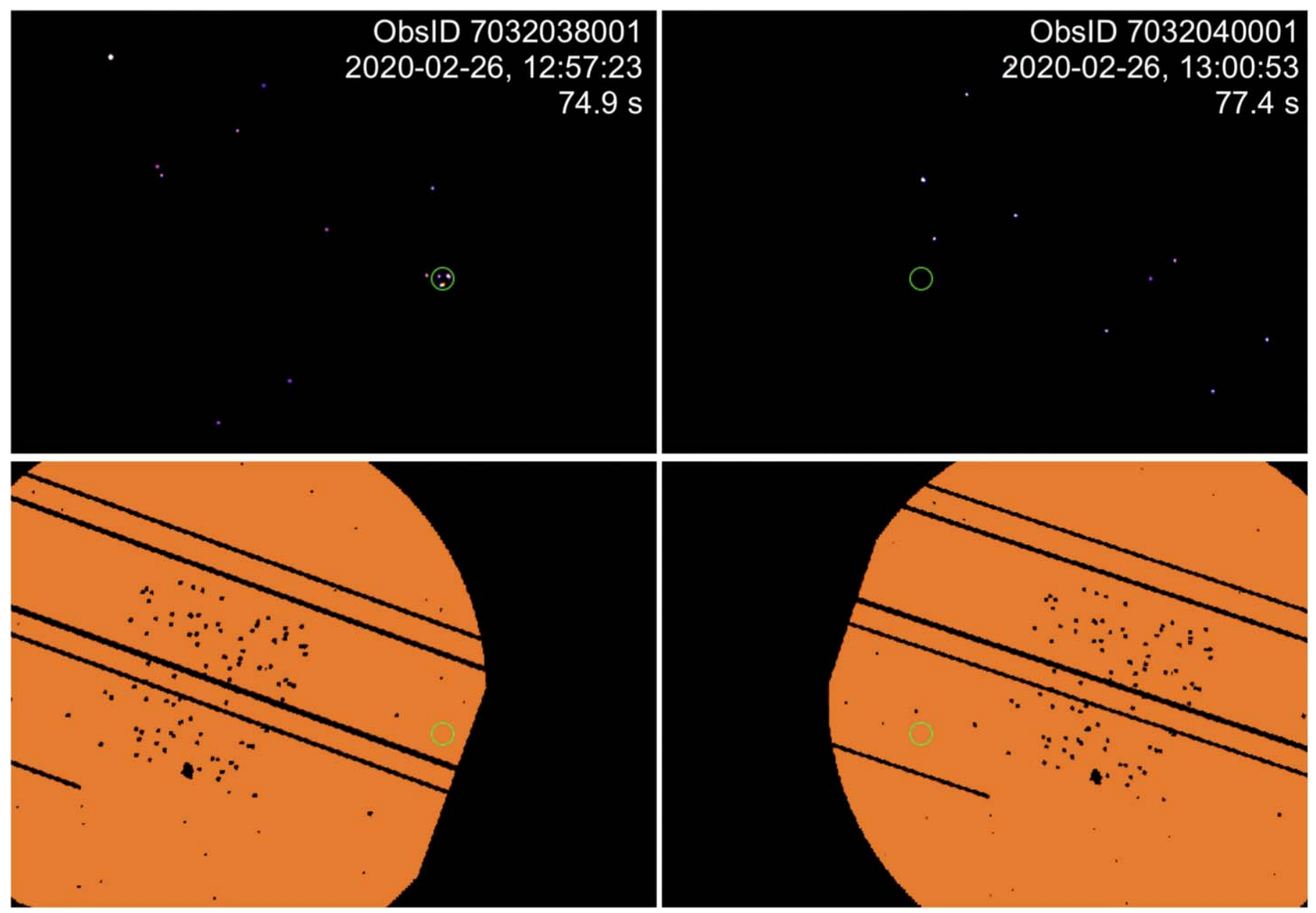

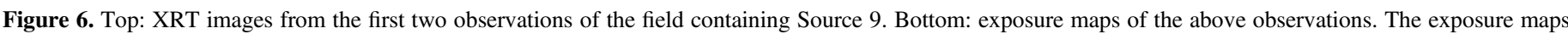
show where the source lies in the XRT FOV, along with the locations of dead pixels and hot columns (which are masked out).

Q1_src40 was found at R.A., decl. $($ J2000 $)=173.27717$, -2.44852 with an estimated uncertainty of $0.8^{\prime \prime}$ (radius, 90\% confidence), starting at $T_{0}+2.13$ days. The initial $u$-band image gave a $3.2 \sigma$ detection with a magnitude of $19.42 \pm 0.34$ (20.44 mag. AB). A second, longer UVOT image (258 s) gave a $5.5 \sigma$ detection with a $u$-band magnitude of $19.54 \pm 0.20$ (20.56 mag. AB) taken at $T_{0}+10.54$ days. A cataloged source, SDSS J113306.49-022654.8, is consistent with the location of this source, and with archival magnitude $u=21.168 \pm 0.081$ (AB). This source is listed in several catalogs as a candidate AGN/quasar (Suchkov et al. 2005; Brescia et al. 2015; Nakoneczny et al. 2019). The observed brightening (compared to the archival magnitude) is likely due to AGN variability. However, if the $\mathrm{BBH}$ merger occurred in an AGN accretion disk, the kicked remnant BH could disturb the disk, making AGN brighten (see, e.g., Graham et al. 2020), although such a scenario cannot be confidently discerned with the available data.

Q1_src54 was identified at an R.A., decl. (J2000) = 173.30543, -2.46977 with an estimated uncertainty of $0.8^{\prime \prime}$ (radius, 90\% confidence), starting at $T_{0}+2.13$ days. The $75 \mathrm{~s}$ image gave a $3.4 \sigma$ detection with a $u$-band magnitude of $19.04 \pm 0.32$ (20.06 mag. AB). The source was not detected in a second, longer UVOT $u$-band image (258s) with a $3 \sigma$ upper limit of $>19.94$ taken at $T_{0}+10.54$ days, suggesting the source has faded between observations. A deeper (905 s) $u$-band observation taken at $T_{0}+136$ days failed to find any source, down to a magnitude of $>20.44$ ( $>21.46$ AB). Q1_src54 was found to have a WISE counterpart whose position is consistent within $4^{\prime \prime}$, which may suggest an underlying red progenitor, potentially associating this $u$-band detection as a possible stellar flare from a cool star (for details, see S. R. Oates et al. (2020, in preparation)).

\section{Physical Implications}

No credible or likely EM counterpart to S200224ca was detected in our follow-up XRT/UVOT observations, in the BAT data around the time of trigger, or by other observatories. The lack of detection of any candidate counterparts, though, is a confirmation of the expected (null) result, or that emission (if present) was below the sensitivity threshold for the UVOT/ $\mathrm{XRT} / \mathrm{BAT}$, as well as for those of the other observatories that followed up on this event.

With the trigger distance, $d=1575 \pm 322 \mathrm{Mpc}$, the $\sim 4.76 \times$ $10^{-7} \mathrm{erg} \mathrm{cm}^{-2} \mathrm{~s}^{-1}$ BAT upper limit (in the $15-350 \mathrm{keV}$ band) corresponds to a gamma-ray luminosity upper limit of $1.4_{-0.5}^{+0.6} \times$ $10^{50} \mathrm{erg} \mathrm{s}^{-1}$. In $80 \mathrm{~s}$ exposures, the XRT reaches a sensitivity of $\approx 4.2 \times 10^{-12}$ erg cm $\mathrm{cm}^{-2} \mathrm{~s}^{-1}$ at $50 \%$ confidence, and $\approx$ $10^{-11} \mathrm{erg} \mathrm{cm}^{-2} \mathrm{~s}^{-1}$ at $90 \%$ confidence $(0.3-10 \mathrm{keV})$. These correspond to luminosity upper limits of $1.2_{-0.4}^{+0.6} \times 10^{45}$ and $3.0_{-1.1}^{+1.3} \times 10^{45} \mathrm{erg} \mathrm{s}^{-1}$, respectively, at the trigger distance. With the same exposures, the UVOT can reach an approximate $u$-band limiting magnitude of 19.2 (Vega), or 20.2 (AB). This corresponds 
to a flux density of $<7.04 \times 10^{-17} \mathrm{erg} \mathrm{cm}^{-2} \mathrm{~s}^{-1} \AA^{-1}$ (at $3465 \AA$, the center of UVOT's $u$-filter), and a luminosity $\left(\nu L_{\nu}\right)$ upper limit of $7.2_{-2.7}^{+3.3} \times 10^{43} \mathrm{erg} \mathrm{s}^{-1}$ (at $8.65 \times 10^{14} \mathrm{~Hz}$ ). The uncertainties on the luminosity upper limits correspond to the uncertainty in estimated merger distance.

It is worth noting that UVOT's upper limits are not as constraining as those provided by larger, ground-based optical observatories that covered the event, although UVOT's limits are at bluer wavelengths. For example, Subaru-HSC covered $83.7 \%$ of the GW error region between $T_{0}+12.3 \mathrm{hr}$ and $T_{0}+13.4 \mathrm{hr}$, and obtained limiting $(\mathrm{AB})$ magnitudes in the $r$ - and $z$-band of $>24.8$ and $>23.5$, respectively (Ohgami et al. 2020). These Subaru limits correspond to flux densities of $<3.38 \times 10^{-19} \mathrm{erg}$ $\mathrm{cm}^{-2} \mathrm{~s}^{-1} \AA^{-1}$ (at $6222 \AA$ ) and $<5.43 \times 10^{-19} \mathrm{erg} \mathrm{cm}^{-2} \mathrm{~s}^{-1} \AA^{-1}$ (at $8917 \AA$ ). These tighter flux density upper limits place luminosity $\left(\nu L_{\nu}\right)$ upper limits $6.2_{-2.2}^{+2.7} \times 10^{41} \mathrm{erg} \mathrm{s}^{-1}$ at $4.82 \times$ $10^{14} \mathrm{~Hz}$, and $1.43_{-0.52}^{+0.65} \times 10^{42} \mathrm{erg} \mathrm{s}^{-1}$ at $3.36 \times 10^{14} \mathrm{~Hz}$.

These upper limits place some constraints on the proposed models for electromagnetic counterparts of $\mathrm{BBH}$ mergers. The prompt emission luminosity upper limit (from the BAT) is about one order of magnitude higher than the claimed luminosity of GW150914-GBM (Connaughton et al. 2017), so this upper limit is not very constraining for prompt GRB models for BBH mergers (Loeb 2016; Perna et al. 2016; Zhang 2016). Nevertheless, one may constrain the upper limit of the dimensionless charge $\hat{q}$ of the BHs. Using Equation (11) of Zhang (2019) ${ }^{36}$ and assuming that both BHs have roughly the same mass and $\hat{q}$ (noting $a=2 r_{s}$ at the merger, where $r_{s}$ is the Schwarzschild radius), one can derive $\hat{q}^{2}\left(c^{5} / G\right) / 48<1.4 \times 10^{50} \mathrm{erg} \mathrm{s}^{-1}$, which gives a shallow upper limit on the dimensionless charge

$$
\hat{q}<1.4 \times 10^{-4}
$$

This upper limit is higher than the value required to interpret GW150914-GBM, $\hat{q} \sim 5 \times 10^{-5}$, which has been regarded as extremely large for stellar-mass black holes (Zhang 2016). Therefore, this upper limit is not very constraining. The nondetection of bright gamma-rays at the merger time also disfavors bright emission from extreme scenarios, such as forming two black holes in a collapsing star (Loeb 2016) or reactivating a massive dead disk right after the merger (Perna et al. 2016), both requiring quite specific physical conditions.

The X-ray upper limit can place an upper limit on the total energy of a putative electromagnetic explosion associated with the BBH merger event. Using Equation (20) of Wang et al. (2015), with an X-ray flux upper limit of $4.2 \times 10^{-12} \mathrm{erg} \mathrm{cm}^{-2} \mathrm{~s}^{-1}$ at $22 \mathrm{ks}$ after the merger, ${ }^{37}$ one can obtain an upper limit on the isotropic-equivalent energy of the blast wave (see also Perna et al. 2019)

$$
E<4.1 \times 10^{51} \mathrm{erg}
$$

\footnotetext{
36 We only consider the electric dipole radiation luminosity and neglect the magnetic dipole radiation luminosity (Equation (17) of Zhang 2019) to constrain $\hat{q}$. This is because the $a$-dependence is very steep for the latter component. Even if the instantaneous luminosities are comparable for the two components at the merger time, the total emitted energy from the latter is much smaller than the former. Therefore, it can be neglected when the average luminosity is considered for comparison with the luminosity upper limit from observations.

37 The luminosity upper limit corresponds to an $80 \mathrm{~s}$ XRT exposure. Since the constraint on $E$ is tighter at earlier epochs, we adopt the earliest epoch, $22 \mathrm{ks}$ (post-merger), in the calculation in order to reach the most stringent constraint on $E$.
}

assuming typical GRB parameters: electron energy distribution power-law index $p=2.2$, electron energy fraction $\epsilon_{e}=0.1$, magnetic energy fraction $\epsilon_{B}=0.01$, and circumburst number density $n=1 \mathrm{~cm}^{-3}$ for the source. ${ }^{38}$ This rules out a bright GRB-like explosion, but a relatively faint explosion (e.g., a low-luminosity GRB) is not ruled out. For comparison, the isotropic-equivalent energy released by GRB 170817A (GW170817) was estimated to be $E \sim 1 \times 10^{53} \mathrm{erg}$.

Finally, the peak flux of the optical flare peaking $\sim 50$ days after GW190521g (another BBH merger) is $\sim 10^{45} \mathrm{erg} \mathrm{s}^{-1}$ (Graham et al. 2020). The optical/near-UV upper limits we have derived here for S200224ca (from the Subaru/HSC coverage) are lower by at least two orders of magnitudes, even though the time of follow-up observations was much earlier than 50 days. This disfavors the AGN disk interaction model for an early interaction (assuming, of course, that the location of the putative optical counterpart to S200224ca was observed).

\section{Summary}

During its third observing run, LVC detected S200224ca: a candidate GW event with a low false-alarm rate (roughly once every $1974 \mathrm{yr}$ ) and a low probability of being terrestrial $\left(P_{\text {Terrestrial }}=3.3952 \times 10^{-5}\right)$. This trigger was confidently identified to be produced by a $\mathrm{BBH}$ merger at an estimated distance $d=1575 \pm 322 \mathrm{Mpc}$. The event was very welllocalized, with $50 \%$ and $90 \%$ probability areas corresponding to 17 and $69 \mathrm{deg}^{2}$, respectively.

At $T_{0}$, the Swift-BAT FOV covered $88.38 \%$ of the integrated GW localization region (with partial coding fraction $>10 \%$ ). No significant $(\gtrsim 5 \sigma)$ detections were seen in the BAT raw light curves. Using the light curve with $1.6 \mathrm{~s}$ bins, we place a $5 \sigma$ upper flux limit of $<4.76 \times 10^{-7} \mathrm{erg} \mathrm{cm}^{-2} \mathrm{~s}^{-1}$ (in $15-350 \mathrm{keV}$ ) on prompt emission from the merger within $T_{0} \pm 100 \mathrm{~s}$. No significant detections were seen in the survey data either (data binned to intervals of $\sim 300 \mathrm{~s}$ ), between $T_{0}-115$ to $T_{0}-22 \mathrm{~s}$, and $T_{0}+180$ to $T_{0}+480 \mathrm{~s}$. Looking forward, however, upon notice of GW triggers, the newly implemented BAT GUANO system (Tohuvavohu et al. 2020) will continue to attempt to automatically retrieve event data around trigger times. This will allow for much more sensitive searches for prompt (sGRB-like) emission from $\mathrm{CBC}$ events, and will also increase the number of possible co-detections of GW and EM radiation. Currently, the GUANO event recovery success rate has now reached $\sim 90 \%$.

Swift performed targeted follow-up observations of the GW error region with the XRT and UVOT from $T_{0}+22 \mathrm{ks}$ to $T_{0}+196.5 \mathrm{ks}$. The XRT and UVOT observed 672 fields for approximately $80 \mathrm{~s}$ each. The XRT covered $64.5 \mathrm{deg}^{2}$, corresponding to approximately $80 \%$ of the galaxy-convolved (and $82 \%$ of the raw) probability region, making S200224ca the $\mathrm{BBH}$ event most thoroughly followed-up in X-rays to date.

Although no likely EM counterparts were detected by Swift BAT, XRT, or UVOT, nor by other facilities in any wavelength, these searches serve as observational evidence supporting the expected (null) result. From the BAT data, we place a gamma-ray $(15-350 \mathrm{keV})$ luminosity upper limit of $1.4_{-0.5}^{+0.6} \times 10^{50} \mathrm{erg} \mathrm{s}^{-1}$ on $\mathrm{S} 200224 \mathrm{ca}$ at $T_{0} \pm 100 \mathrm{~s}$. From the XRT observations, we place X-ray $(0.3-10 \mathrm{keV})$ luminosity upper limits of $1.2_{-0.4}^{+0.6} \times 10^{45}$ and $3.0_{-1.1}^{+1.3} \times 10^{45} \mathrm{erg} \mathrm{s}^{-1}$ (at $50 \%$ and $90 \%$ confidence) on

\footnotetext{
38 This estimate assumed $\nu_{m}<\nu<\nu_{c}$, which has a dependence of $n^{-2 /(p+3)}$. For $\nu>\nu_{c}$, the upper limit is similar, but the result does not depend on $n$.
} 
S200224ca from $T_{0}+22 \mathrm{ks}$ to $T_{0}+196.5 \mathrm{ks}$. From the UVOT $u$ band observations, we place a luminosity $\left(\nu L_{\nu}\right)$ upper limit of $7.24 \times 10^{43} \mathrm{erg} \mathrm{s}^{-1}$ (at $8.65 \times 10^{14} \mathrm{~Hz}$ ). We calculate tighter limits (albeit at redder wavelengths) from optical ground-based observations (e.g., Subaru-HSC; Ohgami et al. 2020), which place luminosity $\nu L_{\nu}<6.2_{-2.2}^{+2.7} \times 10^{41} \mathrm{erg} \mathrm{s}^{-1}$ at $4.82 \times 10^{14} \mathrm{~Hz}(r$ band), and $<1.43_{-0.52}^{+0.65} \times 10^{42} \mathrm{erg} \mathrm{s}^{-1}$ at $3.36 \times 10^{14} \mathrm{~Hz}$ (z-band).

From these limits, we place a shallow upper limit on the dimensionless BH charge, $\hat{q}<1.4 \times 10^{-4}$, and an upper limit on the isotropic-equivalent energy of a blast wave from the merger $E<4.1 \times 10^{51} \mathrm{erg}$ (assuming typical GRB parameters). These limits also disfavor bright GRB-like emission from the BBH merger, but still allow for faint emission (e.g., a lowluminosity GRB). Finally, the nondetection of gamma-rays disfavors some hypothetical exotic scenarios, such as the formation of two black holes in a collapsing star (Loeb 2016), reactivating a massive dead disk right after the merger (Perna et al. 2016), or BHs with an extremely large charge in excess of $\hat{q}=1.4 \times 10^{-4}$.

This publication is an official product of the Swift GW follow-up team. The authors wish to thank the anonymous referee for helpful comments that enhanced the clarity of the paper. N. J. K. and J. A. K. would like to acknowledge support from NASA Grants 80NSSC19K0408 and 80NSSC20K1104. P. A. E., K. L. P., A. P. B., J. P. O., A. A. B., S. W. K. E., N. P. M. K., and M. J. P. acknowledge funding from the UK Space Agency. P. D. A. acknowledges support from PRIN-MIUR 2017 (grant 20179ZF5KS). M. G. B., G. C., S. C., A. D. A., P. D. A., A. M. and G. T. acknowledge funding from the Italian Space Agency, contract ASI/INAF n. I/004/11/4. The authors would like to acknowledge support from the Italian Ministry of Foreign Affairs and International Cooperation grant MAE0065741. E. T. acknowledges support by the National Aeronautics and Space Administration through grant NNX10AF62G issued through the Astrophysics Data Analysis Program. M. D. P. acknowledges support for this work by the Scientific and Technological Research Council of Turkey (TÜBİTAK), grant No: MFAG-119F073. D. B. M. is supported by research grant 19054 from Villum Fonden.

Facility: NASA Neil Gehrels Swift Observatory.

\section{ORCID iDs}

N. J. Klingler (1D https://orcid.org/0000-0002-7465-0941 S. R. Oates (i) https://orcid.org/0000-0001-9309-7873 J. A. Kennea (i) https://orcid.org/0000-0002-6745-4790 P. A. Evans (iD https://orcid.org/0000-0002-8465-3353

A. Tohuvavohu (10) https://orcid.org/0000-0002-2810-8764

B. Zhang (i) https://orcid.org/0000-0002-9725-2524

K. L. Page (1) https://orcid.org/0000-0001-5624-2613

S. B. Cenko (i) https://orcid.org/0000-0003-1673-970X

M. G. Bernardini (ib https://orcid.org/0000-0001-6106-3046

A. A. Breeveld (1) https://orcid.org/0000-0002-0001-7270

P. J. Brown (1) https://orcid.org/0000-0001-6272-5507

D. N. Burrows (1) https://orcid.org/0000-0003-0729-1632

S. Campana (iD https://orcid.org/0000-0001-6278-1576

A. D’Aì (i) https://orcid.org/0000-0002-5042-1036

V. D'Elia (i) https://orcid.org/0000-0002-7320-5862

J. Garcia (iD https://orcid.org/0000-0003-3828-2448

P. Giommi (1) https://orcid.org/0000-0002-2265-5003

C. Gronwall (i) https://orcid.org/0000-0001-6842-2371

D. H. Hartmann (i) https://orcid.org/0000-0002-8028-0991
N. P. M. Kuin (1D https://orcid.org/0000-0003-4650-4186

D. B. Malesani (i) https://orcid.org/0000-0002-7517-326X

F. E. Marshall (i) https://orcid.org/0000-0002-3559-6305

P. T. O'Brien (Di) https://orcid.org/0000-0002-5128-1899

J. P. Osborne (iD https://orcid.org/0000-0002-1041-7542

D. M. Palmer (iD https://orcid.org/0000-0001-7128-0802

M. J. Page (iD https://orcid.org/0000-0002-6689-6271

M. Perri (1) https://orcid.org/0000-0003-3613-4409

J. L. Racusin (iD https://orcid.org/0000-0002-4744-9898

T. Sakamoto (iD https://orcid.org/0000-0001-6276-6616

B. Sbarufatti (iD https://orcid.org/0000-0001-6620-8347

J. E. Schlieder (iD https://orcid.org/0000-0001-5347-7062

G. Tagliaferri (iD https://orcid.org/0000-0003-0121-0723

E. Troja (iD https://orcid.org/0000-0002-1869-7817

\section{References}

Abbott, B. P., Abbott, R., Abbott, T. D., et al. 2016a, ApJS, 225, 8 Abbott, B. P., Abbott, R., Abbott, T. D., et al. 2016b, PhRvL, 116, 241103 Abbott, B. P., Abbott, R., Abbott, T. D., et al. 2016c, PhRvX, 6, 041015 Abbott, B. P., Abbott, R., Abbott, T. D., et al. 2017a, ApJL, 848, L12 Abbott, B. P., Abbott, R., Abbott, T. D., et al. 2017b, ApJL, 848, L13 Abbott, B. P., Abbott, R., Abbott, T. D., et al. 2019a, PhRvX, 9, 031040 Abbott, B. P., Abbott, R., Abbott, T. D., et al. 2019b, ApJL, 882, L24 Abbott, R., Abbott, T. D., Abraham, S., et al. 2020, arXiv:2010.14527 Acernese, F., Agathos, M., Agatsuma, K., et al. 2015, CQGra, 32, 024001 Bailyn, C. D., Jain, R. K., Coppi, P., et al. 1998, ApJ, 499, 367 Barthelmy, S. D., Barbier, L. M., Cummings, J. R., et al. 2005, SSRv, 120, 143 Barthelmy, S. D., Lien, A. Y., Palmer, D. M., et al. 2020, GCN Circ. 27216, https://gcn.gsfc.nasa.gov/gcn3/27216.gcn3

Bertin, E., \& Arnouts, S. 1996, A\&AS, 117, 393

Bilicki, M., Jarrett, T. H., Peacock, J. A., Cluver, M. E., \& Steward, L. 2014, ApJS, 210, 9

Breeveld, A. A., Oates, S. R., Marshall, F. E., et al. 2020, GCN Circ. 27288, https://gcn.gsfc.nasa.gov/gen3/27288.gcn3

Brescia, M., Cavuoti, S., \& Longo, G. 2015, MNRAS, 450, 3893

Burrows, D. N., Hill, J. E., Nousek, J. A., et al. 2005, SSRv, 120, 165

Connaughton, V., Blackburn, L., Briggs, M. S., et al. 2017, GCN Circ. 21506, https://gcn.gsfc.nasa.gov/gcn3/21506.gcn3

Connaughton, V., Burns, E., Goldstein, A., et al. 2016, ApJL, 826, L6 Connaughton, V., Burns, E., Goldstein, A., et al. 2018, ApJL, 853, L9 Dálya, G., Galgóczi, G., Dobos, L., et al. 2018, MNRAS, 479, 2374

Evans, P. A., Cenko, S. B., Kennea, J. A., et al. 2017, Sci, 358, 1565 Evans, P. A., Kennea, J. A., Barthelmy, S. D., et al. 2016a, MNRAS, 460, L40 Evans, P. A., Kennea, J. A., Palmer, D. M., et al. 2016b, MNRAS, 462, 1591 Evans, P. A., Kennea, J. A., Palmer, D. M., et al. 2019, MNRAS, 484, 2362 Evans, P. A., Osborne, J. P., Kennea, J. A., et al. 2015, MNRAS, 448, 2210 Evans, P. A., Osborne, J. P., Kennea, J. A., et al. 2016c, MNRAS, 455, 1522 Evans, P. A., Page, K. L., Osborne, J. P., et al. 2020, ApJS, 247, 54

Fong, W., Berger, E., Margutti, R., \& Zauderer, B. A. 2015, ApJ, 815, 102 Gal-Yam, A. 2012, Sci, 337, 927

Gehrels, N., Chincarini, G., Giommi, P., et al. 2004, ApJ, 611, 1005 Goldstein, A., Veres, P., Burns, E., et al. 2017, ApJL, 848, L14

Graham, M. J., Ford, K. E. S., McKernan, B., et al. 2020, PhRvL, 124, 251102 Greiner, J., Burgess, J. M., Savchenko, V., et al. 2016, ApJL, 827, L38

Hallinan, G., Corsi, A., Mooley, K. P., et al. 2017, Sci, 358, 1579

Kamble, A., \& Kaplan, D. L. A. 2013, IJMPD, 22, 1341011

Klingler, N. J., Kennea, J. A., Evans, P. A., et al. 2019, ApJS, 245, 15

Lien, A., Sakamoto, T., Barthelmy, S. D., et al. 2016, ApJ, 829, 7

Lien, A., Sakamoto, T., Gehrels, N., et al. 2014, ApJ, 783, 24

LIGO Scientific Collaboration, Aasi, J., Abbott, B. P., et al. 2015, CQGra, 32, 074001

LIGO Scientific Collaboration \& Virgo Collaboration 2020a, GCN Circ. 27184, https://gcn.gsfc.nasa.gov/gcn3/27184.gcn3

LIGO Scientific Collaboration \& Virgo Collaboration 2020b, GCN Circ. 27262, https://gcn.gsfc.nasa.gov/gcn3/27262.gcn3

Liu, T., Romero, G. E., Liu, M.-L., \& Li, A. 2016, ApJ, 826, 82

Loeb, A. 2016, ApJL, 819, L21

MacLeod, C. L., Ivezić, Ž., Sesar, B., et al. 2012, ApJ, 753, 106

Martin, R. G., Nixon, C., Xie, F.-G., et al. 2018, MNRAS, 480, 4732

McKernan, B., Ford, K. E. S., Bartos, I., et al. 2019, ApJL, 884, L50 Metzger, B. D. 2019, LRR, 23, 1

Nakoneczny, S., Bilicki, M., Solarz, A., et al. 2019, A\&A, 624, A13 
Ohgami, T., Tominaga, N., Morokuma, T., et al. 2020, GCN Circ. 27205, https://gcn.gsfc.nasa.gov/gen3/27205.gen3

Osten, R. A., Godet, O., Drake, S., et al. 2010, ApJ, 721, 785

Özel, F., \& Freire, P. 2016, ARA\&A, 54, 401

Özel, F., Psaltis, D., Narayan, R., et al. 2010, ApJ, 725, 1918

Page, K. L., Evans, P. A., Tohuvavohu, A., et al. 2020, MNRAS, 499, 3459

Perna, R., Lazzati, D., \& Farr, W. 2019, ApJ, 875, 49

Perna, R., Lazzati, D., \& Giacomazzo, B. 2016, ApJL, 821, L18

Roming, P. W. A., Kennedy, T. E., Mason, K. O., et al. 2005, SSRv, 120, 95

Sari, R., Piran, T., \& Narayan, R. 1998, ApJL, 497, L17

Savchenko, V., Mereghetti, S., Ferringo, C., et al. 2017, GCN Circ. 21507, https://gcn.gsfc.nasa.gov/gcn3/21507.gcn3

Schmidt, S. J., Prieto, J. L., Stanek, K. Z., et al. 2014, ApJL, 781, L24
Smith, K. L., Mushotzky, R. F., Boyd, P. T., et al. 2018, ApJ, 857, 141 Suchkov, A. A., Hanisch, R. J., \& Margon, B. 2005, AJ, 130, 2439

Tohuvavohu, A., Kennea, J. A., DeLaunay, J., et al. 2020, ApJ, 900, 35

Troja, E., Piro, L., van Eerten, H., et al. 2017, Natur, 551, 71

van Velzen, S., Gezari, S., Hammerstein, E., et al. 2020, arXiv:2001.01409

Veres, P., Canton, T. D., Burns, E., et al. 2019, ApJ, 882, 53

Wanderman, D., \& Piran, T. 2015, MNRAS, 448, 3026

Wang, X.-G., Zhang, B., Liang, E.-W., et al. 2015, ApJS, 219, 9

Wheeler, J. C., \& Harkness, R. P. 1990, RPPh, 53, 1467

Wyatt, S. D., Tohuvavohu, A., Arcavi, I., et al. 2020, ApJ, 894, 127

Yamazaki, R., Asano, K., \& Ohira, Y. 2016, PTEP, 2016, 051E01

Zhang, B. 2016, ApJL, 827, L31

Zhang, B. 2019, ApJL, 873, L9 